\title{
On Some Mixed Trilateral Generating Functions of Modified Jacobi Polynomials by Group Theoretic Method
}

\author{
S. Alam*, A. K. Chongdar \\ Department of Mathematics, Bengal Engineering and Science University, Shibpur P.O. Botanic Garden, Howrah - 711 103, India \\ *Corresponding Author: salam50in@yahoo.co.in
}

Copyright (C2013 Horizon Research Publishing All rights reserved.

\begin{abstract}
In this note, we have obtained some novel results on mixed trilateral generating functions involving $P_{n}^{(\alpha+n, \beta)}(x)$, a modification of Jacobi polynomials by group-theoretic method. We have introduced a linear partial differential operator and found the corresponding extended form of the group. Finally, we obtained a novel generating function with the help of which, our desired result has been established.
\end{abstract}

Keywords Trilateral generating relation, Jacobi polynomial, Special functions

\section{Introduction}

Generating functions play a large role in the study of special functions. Generating functions which are available in the literature are almost bilateral in nature. There is a dearth of trilateral generating functions in the field of special functions. Group-theoretic method of obtaining generating functions for various special functions has been receiving much attention in recent years. In fact, the idea of group-theoretic method in the study of generating functions of various special functions started in the middle of the last century by L. Weisner[1] while investigating generating functions of Hypergeometric functions. From seventies and onwards (i.e. just after the publication of the book "Obtaining generating functions" by E. B. McBride[2]) of the last century, Weisner's method has been extensively utilized by researchers in the derivation of generating functions of various special functions. In the present article, we have adopted group theoretic method to obtain 
a novel result on mixed trilateral generating functions of modified Jacobi polynomials, $P_{n}^{(\alpha+n, \beta)}(x)$ where $P_{n}^{(\alpha, \beta)}(x)$ is defined by [3]:

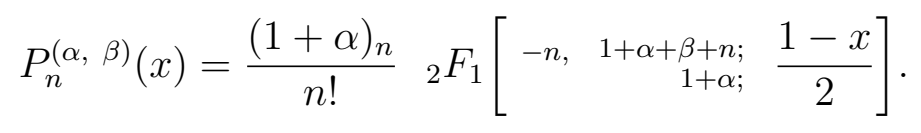

The main result of our investigation is stated in the form of the following theorem. For previous works on trilateral generating functions of Jacobi / modified Jacobi polynomials by group theoretic method, one can see the works [4-6].

Theorem-I: If there exists a bilateral generating relation of the form:

$$
G(x, u, t)=\sum_{n=0}^{\infty} a_{n} P_{n}^{(\alpha+n, \beta)}(x) g_{n}(u) t^{n}
$$

where $g_{n}(u)$ is an arbitrary polynomial of degree $\mathrm{n}$, then

$$
\begin{aligned}
& (1+t)^{\alpha}\left[1+\frac{t}{2}(1-x)\right]^{-\alpha-\beta-1} G\left(\frac{x-\frac{t}{2}(1-x)}{1+\frac{t}{2}(1-x)}, u, \frac{t z(1+t)}{\left[1+\frac{t}{2}(1-x)\right]^{2}}\right) \\
& =\sum_{n=0}^{\infty} \sigma_{n}(x, u, t) z^{n},
\end{aligned}
$$

where

$$
\sigma_{n}(x, u, t)=\sum_{p=0}^{n} a_{p}\left(\begin{array}{l}
n \\
p
\end{array}\right) P_{n}^{(\alpha-n+2 p, \beta)}(x) g_{p}(u) t^{p}
$$

To prove the theorem, we introduce a linear partial differential operator and the corresponding extended form of the group in the next section. Finally, as an application of the operator, we shall obtain a novel generating relation of the special function under consideration with the help of which Theorem-1 will be proved. 


\section{Derivation of the operator, its extended form of the group and generating function.}

\subsection{Derivation of the operator}

At first we seek the following first order linear partial differential operator:

$$
R=A_{1} \frac{\partial}{\partial x}+A_{2} \frac{\partial}{\partial y}+A_{3} \frac{\partial}{\partial z}+A_{0}
$$

such that

$$
R\left(P_{n}^{(\alpha+n, \beta)}(x) y^{\alpha} z^{n}\right)=a_{n} P_{n+1}^{(\alpha+n-1, \beta)}(x) y^{\alpha-2} z^{n+1}
$$

where $A_{i}(i=0,1,2,3)$ are functions of $x, y, z$ but independent of $\mathrm{n}, \alpha$ and $a_{n}$ is a function of $\mathrm{n}$, $\alpha, \beta$ but independent of $x, y, z$.

Now using the following differential recurrence relation [3]:

$$
\begin{aligned}
\frac{d}{d x}\left(P_{n}^{(\alpha, \beta)}(x)\right)= & \frac{1}{1-x^{2}}[(n+\alpha+\beta+1)(x-1)+2 \alpha] P_{n}^{(\alpha, \beta)}(x) \\
& -2(n+1) P_{n+1}^{(\alpha-1, \beta)}(x),
\end{aligned}
$$

we obtain,

$$
R=\left(1-x^{2}\right) y^{-2} z \frac{\partial}{\partial x}-(x+1) y^{-1} z \frac{\partial}{\partial y}-2 x y^{-2} z^{2} \frac{\partial}{\partial z}-(1+\beta)(x-1) y^{-2} z
$$

such that

$$
R\left(P_{n}^{(\alpha+n, \beta)}(x) y^{\alpha} z^{n}\right)=-2(n+1) P_{n+1}^{(\alpha+n-1, \beta)}(x) y^{\alpha-2} z^{n+1}
$$

\subsection{Extended form of the group generated by $R$}

Now we find the extended form of the group generated by $R$ i.e., we shall find $e^{R} f(x, y, z)$, where $f(x, y, z)$ is arbitrary function and $a$ is an arbitrary constant, real or complex. 
Let $\phi(x, y, z)$ be a function such that $R \phi=0$. Then on solving $R \phi=0$, we get a solution as $\phi=(1+x)^{-\beta} y z^{-1}$. Let us transform $R$ to $E$ where

$$
E=\left(1-x^{2}\right) y^{-2} z \frac{\partial}{\partial x}-(x+1) y^{-1} z \frac{\partial}{\partial y}-2 x y^{-2} z^{2} \frac{\partial}{\partial z}
$$

then

$$
E=\phi^{-1}(x, y, z) R \phi(x, y, z)
$$

i.e,

$$
R=\phi(x, y, z) E \phi^{-1}(x, y, z)
$$

Now let $X, Y, Z$ be a set of new variables for which

$$
E X=1, \quad E Y=0, \quad E Z=0,
$$

so that $E$ reduces to $D=\frac{\partial}{\partial X}$.

Now solving (2.7), we get a set of solutions as follows:

$$
X=\frac{y^{2}}{(x-1) z}, \quad Y=\frac{1-x}{y}, \quad Z=\frac{1-x^{2}}{z}
$$

from which we get

$$
\begin{gathered}
x=-\left(\frac{Z}{X Y^{2}}+1\right) \\
y=\left(\frac{2 X Y^{2}+Z}{X Y^{3}}\right) \\
z=-\left(\frac{Z}{X^{2} Y^{4}}+\frac{2}{X Y^{2}}\right) .
\end{gathered}
$$

Then

$$
\begin{aligned}
& e^{a R} f(x, y, z) \\
& =\phi(x, y, z) e^{a E}\left(\phi^{-1}(x, y, z) f(x, y, z)\right) \\
& =\phi(x, y, z) e^{a D} g(X, Y, Z) \\
& =\phi(x, y, z) g(X+a, Y, Z) .
\end{aligned}
$$


On calculation, we have

$$
\begin{aligned}
& e^{w A} f(x, y, z)=\left[1-(1-x) y^{-2} z w\right]^{-\beta-1} \\
& \quad \times f\left[\frac{x+(1-x) y^{-2} z w}{1-(1-x) y^{-2} z w}, \frac{y\left(1-2 y^{-2} z w\right)}{1-(1-x) y^{-2} z w}, \frac{z\left(1-2 y^{-2} z w\right)}{\left\{1-(1-x) y^{2} z w\right\}^{2}}\right] .
\end{aligned}
$$

\subsection{Application of the operator $R$}

From (2.13), we notice that

$$
\begin{gathered}
e^{w R}\left(P_{n}^{(\alpha+n, \beta)}(x) y^{\alpha} z^{n}\right) \\
=y^{\alpha} z^{n}\left(1-2 y^{-2} z w\right)^{\alpha+n}\left[1-(1-x) y^{-2} z w\right]^{-1-\alpha-\beta-2 n} \\
\times P_{n}^{(\alpha+n, \beta)}\left(\frac{x+(1-x) y^{-2} z w}{1-(1-x) y^{-2} z w}\right) .
\end{gathered}
$$

But by using (2.5), we get

$$
\begin{aligned}
& e^{w R}\left(P_{n}^{(\alpha+n, \beta)}(x) y^{\alpha} z^{n}\right) \\
& =y^{\alpha} z^{n} \sum_{p=0}^{\infty} \frac{\left(-2 a y^{-2} z\right)^{p}}{p !}(n+1)_{p} P_{n+p}^{(\alpha+n-p, \beta)}(x) .
\end{aligned}
$$

Equating (2.14) and (2.15) and then replacing $-2 y^{2} z w$ by $t$, we get

$$
\begin{aligned}
& (1+t)^{\alpha+n}\left[1+\frac{t}{2}(1-x)\right]^{-\alpha-\beta-2 n-1} P_{n}^{(\alpha+n, \beta)}\left(\frac{x-(1-x) \frac{t}{2}}{1+(1-x) \frac{t}{2}}\right) \\
& =\sum_{p=0}^{\infty} \frac{(n+1)_{p}}{p !} P_{n+p}^{(\alpha+n-p, \beta)}(x) t^{p},
\end{aligned}
$$

the above generating function is worthy of notice.

\section{Proof of the Theorem}

We shall now prove the Theorem-1 stated above by using the generating relation (2.16). 
Now the right hand side of (1.3)

$$
\begin{aligned}
& =\sum_{n=0}^{\infty} z^{n} \sigma_{n}(x, t) \\
& =\sum_{n=0}^{\infty} z^{n} \sum_{p=0}^{n} a_{p}\left(\begin{array}{c}
n \\
p
\end{array}\right) P_{n}^{(\alpha-n+2 p, \beta)}(x) g_{p}(u) t^{p} \\
& \text { [ using (1.4) ] } \\
& =\sum_{n=0}^{\infty} \sum_{p=0}^{\infty} z^{n+p} a_{p}\left(\begin{array}{c}
n+p \\
p
\end{array}\right) P_{n+p}^{(\alpha+p-n, \beta)}(x) g_{p}(u) t^{p} \\
& =\sum_{p=0}^{\infty} a_{p} g_{p}(u)(t z)^{p} \sum_{n=0}^{\infty}\left(\begin{array}{c}
n+p \\
p
\end{array}\right) P_{n+p}^{(\alpha+p-n, \beta)}(x) z^{n} \\
& =\sum_{p=0}^{\infty} a_{p}(t z)^{p}(1+z)^{\alpha+p}\left[1+\frac{z}{2}(1-x)\right]^{-\alpha-\beta-2 p-1} \\
& \times P_{p}^{(\alpha+p, \beta)}\left(\frac{x-(1-x) \frac{z}{2}}{1+(1-x) \frac{z}{2}}\right) g_{p}(u) \\
& =(1+z)^{\alpha}\left[1+\frac{z}{2}(1-x)\right]^{-\alpha-\beta-1} \sum_{p=0}^{\infty} a_{p}\left(\frac{t z(1+z)}{\left[1+\frac{z}{2}(1-x)\right]^{2}}\right)^{p} \\
& \times P_{p}^{(\alpha+p, \beta)}\left(\frac{x-(1-x) \frac{z}{2}}{1+(1-x) \frac{z}{2}}\right) g_{p}(u) \\
& =(1+z)^{\alpha}\left[1+\frac{z}{2}(1-x)\right]^{-\alpha-\beta-1} \\
& \times G\left(\frac{x-\frac{z}{2}(1-x)}{1+\frac{z}{2}(1-x}, \quad u, \frac{t z(1+z)}{\left[1+\frac{z}{2}(1-x)\right]^{2}}\right), \quad[\operatorname{using}(1.2)]
\end{aligned}
$$

which is Theorem-1.

Here we would like to point it out that Theorem-1 can be proved as follows by the direct application of the operator $R$ by using the method as discussed in [7].

Let us now assume that

$$
G(x, u, t)=\sum_{n=0}^{\infty} a_{n} P_{n}^{(\alpha+n, \beta)}(x) g_{n}(u) t^{n}
$$

where $g_{n}(u)$ is an arbitrary polynomial of degree n. 
Replacing $t$ by $t z$ in (3.2) and then multiplying both sides of the same by $y^{\alpha}$, we get

$$
y^{\alpha} G(x, u, t z)=\sum_{n=0}^{\infty} a_{n}\left(P_{n}^{(\alpha+n, \beta)}(x) y^{\alpha} z^{n}\right) g_{n}(u) t^{n}
$$

Operating $\exp (w R)$ on both sides of (3.3), we get

$$
\begin{aligned}
& \exp (w A)\left(y^{\alpha} G(x, u, t z)\right) \\
& =\exp (w R)\left(\sum_{n=0}^{\infty} a_{n}\left(P_{n}^{(\alpha+n, \beta)}(x) y^{\alpha} z^{n}\right) g_{n}(u) t^{n}\right) .
\end{aligned}
$$

The left hand side of (3.4), with the help of (2.13), becomes

$$
\begin{aligned}
& {\left[1-(1-x) y^{-2} z w\right]^{-\alpha-\beta-1} y^{\alpha}\left(1-2 y^{-2} z w\right)^{\alpha}} \\
& \quad \times G\left(\frac{x+(1-x) y^{-2} z w}{1-(1-x) y^{-2} z w}, u, \quad \frac{t z\left(1-2 y^{-2} z w\right)}{\left\{1-(1-x) y^{-2} z w\right\}^{2}}\right) .
\end{aligned}
$$

The right hand side of (3.4), with the help of (2.5), becomes

$$
\begin{aligned}
& \sum_{n=0}^{\infty} \sum_{p=0}^{\infty} a_{n} t^{n} \frac{w^{p}}{p !} R^{p}\left(P_{n}^{(\alpha+n, \beta)}(x) y^{\alpha} z^{n}\right) g_{n}(u) \\
= & \sum_{n=0}^{\infty} \sum_{p=0}^{\infty} a_{n} t^{n} \frac{w^{p}}{p !}(-2)^{p}(n+1)_{p} P_{n+p}^{(\alpha+n-p, \beta)}(x) y^{\alpha-2 p} z^{n+p} g_{n}(u) \\
= & y^{\alpha} \sum_{n=0}^{\infty} z^{n+p} \sum_{p=0}^{\infty} a_{n} \frac{\left(\frac{-2 w}{y^{2}}\right)^{p}}{p !}(n+1)_{p} P_{n+p}^{(\alpha+n-p, \beta)}(x) g_{p}(u) t^{n} \\
= & y^{\alpha} \sum_{n=0}^{\infty} z^{n} \sum_{p=0}^{n} a_{n-p} \frac{(n-p+1)_{p}}{p !} P_{n}^{(\alpha+n-2 p, \beta)}(x) g_{p}(u) t^{n-p}\left(\frac{-2 w}{y^{2}}\right)^{p} \\
= & y^{\alpha} \sum_{n=0}^{\infty} z^{n} \sum_{p=0}^{n} a_{p} \frac{(p+1)_{n-p}}{(n-p) !} P_{n}^{(\alpha+n-2 p, \beta)}(x) g_{p}(u) t^{n-p}\left(\frac{-2 w}{y^{2}}\right)^{p} .
\end{aligned}
$$


Equating (3.5) and (3.6) we get

$$
\begin{aligned}
& {\left[1-(1-x) y^{-2} z w\right]^{-\alpha-\beta-1} y^{\alpha}\left(1-2 y^{-2} z w\right)^{\alpha}} \\
& \quad \times G\left(\frac{x+(1-x) y^{-2} z w}{1-(1-x) y^{-2} z w}, u, \frac{t z\left(1-2 y^{-2} z w\right)}{\left\{1-(1-x) y^{-2} z w\right\}^{2}}\right) \\
& =\sum_{n=0}^{\infty} z^{n} \sum_{p=0}^{n} a_{p} \frac{(p+1)_{n-p}}{(n-p) !} P_{n}^{(\alpha+n-2 p, \beta)}(x) g_{n}(u) t^{p}\left(\frac{-2 w}{y^{2}}\right)^{p} .
\end{aligned}
$$

Putting $\frac{-2 w}{y^{2}}=1$ in $(3.7)$, we get

$$
\begin{aligned}
& \left(1+(1-x) \frac{z}{2}\right)^{-\alpha-\beta-1}(1+z)^{\alpha} G\left(\frac{x-(1-x) \frac{z}{2}}{1+(1-x) \frac{z}{2}}, u, \frac{t z(1+z)}{\left\{1+(1-x) \frac{z}{2}\right\}^{2}}\right) \\
& =\sum_{n=0}^{\infty} \sigma_{n}(x, u, t) z^{n},
\end{aligned}
$$

where

$$
\sigma_{n}(x, u, t)=\sum_{p=0}^{n} a_{p}\left(\begin{array}{l}
n \\
p
\end{array}\right) P_{n}^{(\alpha-n+2 p, \beta)}(x) g_{p}(u) t^{p}
$$

This completes the proof of Theorem-1.

Finally, if we use the following symmetry relation

$$
P_{n}^{(\beta, \alpha)}(-x)=(-1)^{n} P_{n}^{(\alpha, \beta)}(x)
$$

we shall get the following result.

Theorem-2: If there exists a bilateral generating relation of the form:

$$
G(x, u, t)=\sum_{n=0}^{\infty} a_{n} P_{n}^{(\alpha, \beta+n)}(x) g_{n}(u) t^{n}
$$

where $g_{n}(u)$ is an arbitrary polynomial of degree $\mathrm{n}$, then 


$$
\begin{aligned}
& (1-z)^{\beta}\left[1-\frac{z}{2}(1+x)\right]^{-\alpha-\beta-1} G\left(\frac{x-\frac{z}{2}(1+x)}{1-\frac{z}{2}(1+x)}, u, \frac{t z(1-z)}{\left[1-\frac{z}{2}(1+x)\right]^{2}}\right) \\
& =\sum_{n=0}^{\infty} z^{n} \sigma_{n}(x, u, t)
\end{aligned}
$$

where

$$
\sigma_{n}(x, u, t)=\sum_{p=0}^{n} a_{p}\left(\begin{array}{c}
n \\
p
\end{array}\right) P_{n}^{(\alpha, \beta-n+2 p)}(x) g_{p}(u) t^{p}
$$

which is analogous to the Theorem-1.

\section{Conclusion}

The importance of the Theorem-1 lies in the fact that whenever one knows a bilateral generating relation (1.2), the corresponding mixed trilateral generating relation can at once be written down from (1.3). Thus one can get a large number of mixed trilateral generating relations from (1.3) by attributing different suitable values to $a_{n}$ in (1.2).

\section{REFERENCES}

[1] Weisner L., Group-theoretic origins of certain generating functions, Pacific J. Math., 5(1955), 1033-1039.

[2] McBride E.B., Obtaining Generating Functions, Springer Verlag, New York, Heidelberg, Berlin, 1971, pp 43-46.

[3] Rainville E.D., Special Functions, Macmillan (1960), New York.

[4] Sen B.K. and Chongdar A.K., On mixed trilateral generating relations involving certain special functions, Indian Journal of Theoretical Physics, 45(2)(1997), 161-168.

[5] Srivastava H.M. and Manocha H.L., A Treatise on Generating Functions, Halsted Press (Ellis Horwood Limited) Chichester(1984), P-132.

[6] Majumder A.B., On trilateral generating functions of Gegenbauer polynomials, Bull. Cal. Math. Soc., 87(1995), 449452 .

[7] Chongdar A.K. and Chatterjea S.K., On class of trilateral generating relations with Tchebycheff polynomials from view point of one parameter group of continuous transformations, Bull. Cal. Math. Soc. 73(1981), 127-140. 